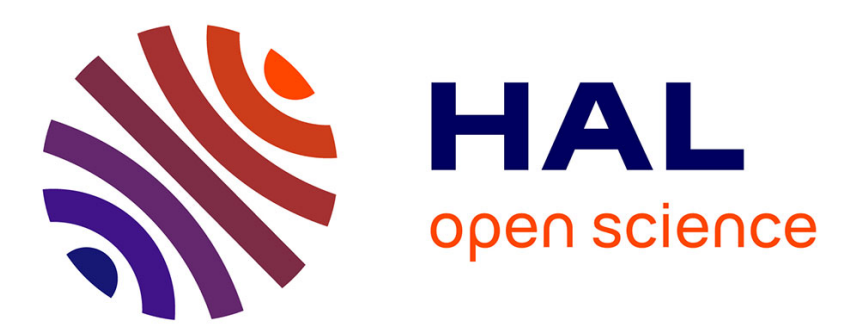

\title{
The neuronal sources of EEG: Modeling of simultaneous scalp and intracerebral recordings in epilepsy.
}

\author{
Delphine Cosandier-Rimélé, Isabelle Merlet, Jean-Michel Badier, Patrick
}

Chauvel, Fabrice Wendling

\section{- To cite this version:}

Delphine Cosandier-Rimélé, Isabelle Merlet, Jean-Michel Badier, Patrick Chauvel, Fabrice Wendling. The neuronal sources of EEG: Modeling of simultaneous scalp and intracerebral recordings in epilepsy.. NeuroImage, 2008, 42 (1), pp.135-46. 10.1016/j.neuroimage.2008.04.185 . inserm-00285582

\section{HAL Id: inserm-00285582 https://www.hal.inserm.fr/inserm-00285582}

Submitted on 5 Jun 2008

HAL is a multi-disciplinary open access archive for the deposit and dissemination of scientific research documents, whether they are published or not. The documents may come from teaching and research institutions in France or abroad, or from public or private research centers.
L'archive ouverte pluridisciplinaire HAL, est destinée au dépôt et à la diffusion de documents scientifiques de niveau recherche, publiés ou non, émanant des établissements d'enseignement et de recherche français ou étrangers, des laboratoires publics ou privés. 


\section{The neuronal sources of EEG: modeling of}

\section{simultaneous scalp and intracerebral}

\section{recordings in epilepsy}

D. Cosandier-Rimélé ${ }^{1,2}$, I. Merlet ${ }^{1,2}$, J.M. Badier ${ }^{3,4}$, P. Chauvel ${ }^{3,4,5}$, F. Wendling ${ }^{1,2}$

${ }^{1}$ INSERM, U642, Rennes, F-35000, France.

${ }^{2}$ Université de Rennes 1, LTSI, Rennes, F-35000, France.

${ }^{3}$ INSERM, U751, Marseille, F-13000, France.

${ }^{4}$ Aix Marseille Université, Faculté de Médecine, Marseille, F-13000, France.

${ }^{5}$ Assistance Publique - Hôpitaux de Marseille, Hôpital de la Timone, Service de Neurophysiologie Clinique, Marseille, F-13000, France.

\section{Correspondence:}

Fabrice Wendling

INSERM U642, Laboratoire Traitement du Signal et de l'Image

Université de Rennes 1

Campus de Beaulieu - Bât. 22

35042 Rennes Cedex, France

E-mail : fabrice.wendling@univ-rennes1.fr 


\section{Abstract}

In many applications which make use of EEG to investigate brain functions, a central question is often to relate the recorded signals to the spatio-temporal organization of the underlying neuronal sources of activity. A modeling attempt to quantitatively investigate this imperfectly known relationship is reported. The proposed plausible model of EEG generation relies on an accurate representation of the neuronal sources of activity. It combines both an anatomically realistic description of the spatial features of the sources (convoluted dipole layer) and a physiologically relevant description of their temporal activities (coupled neuronal populations). The model was used in the particular context of epileptiform activity (interictal spikes) to interpret simultaneously generated scalp and intracerebral EEG. Its integrative properties allowed for the bridging between source-related parameters (spatial extent, location, synchronization) and the properties of resulting EEG signals (amplitude of spikes, amplitude gradient along intracerebral electrodes, topography over scalp electrodes). The sensitivity of both recording modalities to source-related parameters was also studied. The model confirmed that the cortical area involved in interictal spikes is rather large. Its relative location with respect to recording electrodes was found to strongly influence the properties of EEG signals as the source geometry is a critical parameter. The influence, on simulated signals, of the synchronization degree between neuronal populations within the epileptic source was also investigated. The model revealed that intracerebral EEG can reflect epileptic activities corresponding to weak synchronization between neuronal populations of the epileptic patch. These results, as well as the limitations of the model, are discussed.

Keywords: Intracerebral EEG; Scalp EEG; Computational model; Extended source; Spatio-temporal; Forward problem; Epileptic spikes. 


\section{Introduction}

Identifying the neuronal sources of EEG signals has always been considered as a key issue in human neurophysiology (Abraham and Ajmone-Marsan, 1958; Geisler and Gerstein, 1961; Cooper et al., 1965; Gloor, 1985; Ebersole, 1997; Tao et al., 2007b). However, the relationship between the activity generated at the level of neuronal networks and the signals actually recorded from the scalp is not straightforward and still remains imperfectly known. Indeed, the generation of brain electrical activity is a highly complex process resulting from various coupled non-linear mechanisms lying at cellular and network level. Moreover, EEG signals correspond to the projection of neuronal activity onto spatially distributed sensors, and yet they can only partially and macroscopically reflect this activity.

In this paper, we propose a computational modeling approach to better understand the relations between EEG signals and the underlying neuronal sources. Our approach is based on a realistic representation of the sources, as well as the computation of the electrical potentials recorded by electrodes. The present work was carried out in the particular context of epilepsy, in which depth electrode recordings during the presurgical evaluation provide a unique opportunity to record intracerebral EEG activity that can subsequently be confronted to surface EEG. Moreover, the high amplitude and specific waveform of paroxysmal events (epileptic spikes) participate to optimal recording conditions and constitute a favorable framework to such an investigation.

A key feature of our modeling approach is a physiologically relevant representation of the sources of brain electrical activity, which associates a biophysical distributed dipole source model with a biomathematical model of coupled neuronal populations. Dipole models and biologically-inspired neuron models have been both widely used to study the brain activity along with its relationship to the resulting EEG. However, while these two classes of models are usually considered separately, we propose a spatio-temporal extended source model which combines them, in order to achieve the most accurate representation of both the spatial and temporal features of the neuronal sources of activity. 
On the one hand, the current dipole has been widely used to represent the neuronal sources of the EEG. Depending on the spatial scale, dipole models can be classified as point dipole models, in which the neuronal sources are modeled as one or few current dipoles, or distributed dipole models, in which a large number (several thousands) of current dipoles are uniformly distributed within the brain. While point dipole models may reasonably account for focal activity, distributed dipole models are more appropriate for representing extensive activated brain areas. Many distributed dipole models have been proposed in the past decades, most often in the context of EEG source localization (i.e. the estimation of intracerebral sources from measured scalp EEG signals) (Hämäläinen and Ilmoniemi, 1994; Pascual-Marqui et al., 1994; Gorodnitsky et al., 1995; Baillet and Garnero, 1997; Amblard et al., 2004; Daunizeau et al., 2006). Most of them constrain the dipoles to the cortical surface, obtained from MRI segmentation, and allow for a realistic description of the spatial features (spatial extent, 3D geometry) of the sources (Dale and Sereno, 1993). However, such models are generally limited to a simplified description of the temporal features of the sources, with a rather mathematical representation of dipole time-courses.

On the other hand, biologically-inspired neuron models can provide a more accurate description of the temporal activity of neuronal sources (see (Suffczynski et al., 2006) for a detailed review). Depending on the spatial scale, these computational models can be divided into two groups. In microscopic or "detailed" models, single neurons are accurately described and networks are built from the interconnection of a large number (several thousands) of elementary cells (Traub, 1979). Conversely, macroscopic or "lumped" models emphasize the properties of populations of cells instead of individual neurons. Many neurophysiologically relevant macroscopic models have been proposed since the early 1970s (Wilson and Cowan, 1972; Lopes da Silva et al., 1976; Freeman, 1978; Jansen and Rit, 1995). Some of them were shown to be relevant in the context of epilepsy (Wendling et al., 2000, 2005; Suffczynski et al., 2004), as they include parameters that directly relate to mechanisms known to play a crucial role in epileptogenesis, such as excitation, inhibition or coupling degree between neuronal populations.

The spatio-temporal extended source model was used in a previous study to simulate stereoelectroencephalographic (stereo-EEG) signals, as recorded by multicontact intracerebral electrodes in 
epileptic patients (Cosandier-Rimélé et al., 2007). Results showed that the model was able to produce signals that resemble (in terms of morphology and amplitude) to real stereo-EEG signals, for both normal and epileptiform activities. Moreover, in this study, a first attempt was made to investigate the relationship between the recorded intracerebral EEG signals and the underlying neuronal sources. In particular, the quantitative comparison of simulated and real stereo-EEG signals provided insights into the spatial extent and the synchronization level of the activated cortical source during transient epileptiform activities (interictal spikes).

In the present study, we go one step further. From the spatio-temporal extended source model, EEG signals are simulated simultaneously on scalp as well as on intracerebral electrodes. The model is then used to study the influence of several source-related parameters (spatial extent, location, synchronization) on the signals simulated in both modalities. This parametric study is based on several simulation scenarios, each one addressing both qualitatively and quantitatively the specific effect of one parameter on signals.

\section{Methods}

\section{Spatio-temporal modeling of sources}

The architecture of the spatio-temporal extended source model has been described in detail elsewhere (Cosandier-Rimélé et al., 2007). It is based on the combination between a distributed dipole source model (description of the spatial features of the sources) and a model of coupled neuronal populations (simulation of the time-courses associated to the dipole sources) (Fig. 1). A summarized presentation of the source model is given in the sequel.

The sources of the EEG were restricted to the pyramidal neurons of the neocortex. The subcortical structures were not taken into account. Furthermore, it was assumed that the neocortex is organized as a network of neuronal populations. The electrical contribution of each population was represented by a current dipole, characterized by three parameters: its location, its orientation and its intensity. 
Constrained dipole locations and orientations were obtained from a realistic mesh of the neocortical surface (Fig. 1-a). This mesh was built from the segmentation (grey matter/white matter interface) of a three-dimensional magnetic resonance image (3D MRI) (Mangin et al., 1995), using the BrainVisa software (SHFJ, Orsay, France). The resolution of the mesh was high enough to ensure an accurate description of the source space (circumvolutions of the cortical surface). It consisted of 80604 and 83412 triangles for left and right hemisphere, respectively. The average surface of each triangle, corresponding to a distinct population of neurons, was equal to $1 \mathrm{~mm}^{2}$. One dipole was located at the barycenter of each triangle and was oriented normally to its surface (Fig. 1-b).

At each 3D location $\mathbf{x}$, the dipole intensity $I(\mathbf{x}, t)$, function of space and time, was written as the product of two terms:

$$
I(\mathbf{x}, t)=q(\mathbf{x}) \times \alpha(t)
$$

where $q(\mathbf{x})$ denotes the dipole moment (function of space) and $\alpha(t)$ is a weighting coefficient (function of time). The dipole moment $q(\mathbf{x})$ was obtained by multiplying the corresponding triangle surface by the dipole moment surface density of the cerebral cortex. This density was defined as the product of the neocortical thickness (set to a constant value of $3 \mathrm{~mm}$ ) and the cortical current surface density (100 $\mathrm{nA} / \mathrm{mm}^{2}$ for a normal background activity, as described in (Hämäläinen et al., 1993)).

The weighting coefficient $\alpha(t)$ accounts for the time-course of the dipole intensity and is provided by the output of the neuronal population model, which is the sum of average excitatory and inhibitory postsynaptic potentials generated at the level of pyramidal cells. Indeed, it is widely accepted that this postsynaptic activity brings the main contribution to field potentials. As illustrated in Fig. 1-c, each neuronal population contains two subsets of neurons: the main pyramidal cells and the local interneurons. Both subsets mutually interact through excitatory and inhibitory feedback. In each subset, input-output relations are specified by i) a dynamic linear transfer function that changes presynaptic information (i.e. the average density of afferent action potentials) into postsynaptic information (i.e. an average excitatory or inhibitory postsynaptic potential), and by ii) a static nonlinear function that relates the average postsynaptic potential of the subset to an average density of action potentials fired by the neurons. 
Furthermore, projections between pyramidal cells in the neocortex are mainly excitatory. The model of interconnected populations of neurons accounts for this organization by using the average density of action potentials fired by the pyramidal cells of one population as an excitatory input to the pyramidal cells of another population. A connection from a given population $i$ to a population $j$ is characterized by a parameter $K_{i j}$ which represents the degree of coupling. Therefore, an appropriate setting of parameters $K_{i j}$ allows for building specific networks inside which neuronal populations can be unidirectionally and/or bidirectionally coupled (see (Wendling et al., 2000; Wendling and Chauvel, 2008) for details).

Finally, in the model, two conditions (normal and epileptic) are obtained from two different settings of model parameters (excitatory and inhibitory gains in feedback loops, degree and direction of coupling between interconnected populations). These settings are used to simulate the time-course of "focal epileptic sources" (i.e. patches generating interictal spikes) with surrounding normal background activity.

\section{Computation of electrical potentials (forward problem)}

When modeling brain electrical fields, the quasistatic approximation is appropriate, i.e. propagation effects are negligible and there is no time-lag between electrical potentials at any point in the head and the underlying activity (Plonsey and Heppner, 1967). Furthermore, in this study, the sources of the EEG are assumed to be current dipoles with fixed locations and orientations. Therefore, the relationship between sources and electrical potentials measured at sensors can be expressed by the following linear equation

$$
\mathbf{V}=\mathbf{G I}
$$

where $\mathbf{V}$ is the $M \times T$ data matrix (electrical potentials at sensors, relative to a point at infinity), $\mathbf{G}$ is the $M \times N$ gain matrix, and $\mathbf{I}$ is the $N \times T$ matrix of the time-varying intensities of the dipoles ( $M$ : number of sensors, $N$ : number of current dipoles and $T$ : number of time samples). Thus, the computation of the data matrix $\mathbf{V}$ requires the computation of the gain matrix $\mathbf{G}$ and the intensity matrix $\mathbf{I}$. 
The intensity matrix $\mathbf{I}$ is determined by the spatio-temporal source model described above. Each coefficient $I_{i j}$ of $\mathbf{I}$ is obtained from Eq. 1. At each discrete time $t=j(j=1, \ldots, T), I_{i j}$ gives the intensity of the $i$ th dipole $(i=1, \ldots, N)$ at location $\mathbf{x}_{i}$.

The gain matrix $\mathbf{G}$ relates the set of the $N$ current dipoles to the set of the potentials measured at the $M$ sensors. Each coefficient $G_{k i}$ of $\mathbf{G}$ indicates the contribution of the $i$ th dipole (with unit intensity) to the potential at the $k$ th sensor $(k=1, \ldots, M)$. It is obtained by solving the forward problem, i.e. by computing the electrical potential for a given source in the brain. In addition to the dipole location and orientation, and the sensor location, the forward problem solution requires a model of the head. The head model provides a description of the shape and the electrical properties (conductivities) of the different tissues (namely, the brain, the skull and the scalp) in which currents flow. The electrical potentials at any point in the head can then computed from standard physics equations (namely, Poisson's equation).

\section{Computation of electrical potentials on intracerebral electrodes}

To calculate the intracerebral EEG signals, the head was assimilated to a set of three concentric homogeneous spheres representing the brain, the skull and the scalp. Radii of the three spheres were adapted from 3D MRI data, so that the brain tissue was strictly confined inside the innermost sphere. In this study, the radii were set to $84.69 \mathrm{~mm}, 91.62 \mathrm{~mm}$ and $96.13 \mathrm{~mm}$ (brain, skull, scalp, respectively). The conductivity of the skull was assumed to be 40 times lower than that of the brain and scalp, which were set to a same value of $0.33 \mathrm{~S} / \mathrm{m}$ (Gonçalves et al., 2003).

In the spherical model, the forward calculations can be performed at any point in the head volume using an analytical expression (series of Legendre polynomials). In this study, potentials recorded along the intracerebral electrodes were computed as described in (Cosandier-Rimélé et al., 2007) using our own software programs (C language).

In intracerebral recordings, sensors can be spatially close to sources. The classical approximation of the electrical potential due to a current dipole is not valid anymore when the distance between the observation point and the dipole tends to zero. In the model, we checked that potential values computed for the elementary dipoles located in the close vicinity of the intracerebral sensors remain 
finite. In the particular and very rare case where the dipole position is the same as the sensor position, the formula we used to compute potentials (Cosandier-Rimélé et al., 2007) is not defined and the dipole contribution is set to zero.

\section{Computation of electrical potentials on scalp electrodes}

As the locations of scalp electrodes strongly depend on the head shape, a more realistic head model was used to calculate scalp EEG signals. This model consisted in three nested homogeneous compartments (brain, skull and scalp). Surface boundaries between the three tissues were extracted from 3D MRI data (Zanow and Peters, 1995). In this study, surface segmentation and triangulation were performed using the ASA software (ANT, Enschede, Netherlands). Compared to the source model, a lower mesh resolution was sufficient to approximate the shape of volume conductors. Indeed, each mesh (brain, skull and scalp) comprised 2440 triangles. As far as the head conductivity is concerned, assumptions were similar to those made in the case of the spherical model used for the calculation of intracerebral EEG.

In a realistically shaped model, the forward calculations cannot be performed analytically. They must be performed using a numerical method. In this study, the potentials recorded on the scalp surface were computed using the boundary element method (BEM) implemented in the ASA software (ANT, Enschede, Netherlands). This method is based on the isolated problem approach and was initially described in (Hämäläinen and Sarvas, 1989; Meijs et al., 1989).

\section{Model parameter sensitivity analysis}

The model was used to study the influence, on EEG signals recorded by scalp and intracerebral electrodes, of source-related spatio-temporal parameters. Space-dependent parameters included the spatial extent and the location of a focal source of epileptic activity (interictal spikes), as depicted in Fig. 2-a. The studied time-dependent parameter was the synchronization degree within this source. Accordingly, we defined three simulation scenarios, each one addressing the specific influence of one 
parameter. These scenarios were motivated by recurring questions in the context of EEG analysis in epileptic patients. For the sake of clarity, they are detailed just before the presentation of results obtained in each case.

For each scenario, scalp EEG and intracerebral EEG signals were simulated simultaneously. Scalp EEG signals were computed over 63 electrodes distributed on the scalp surface according to the standard 10-10 electrode system (Fig. 2-b). Intracerebral EEG signals were computed at each of the 15 sensors along an intracerebral electrode, orthogonally implanted in the left temporo-parieto-occipital region (Fig. 2-c). Electrode sensors were cylinders (length: $2 \mathrm{~mm}$, diameter: $0.8 \mathrm{~mm}$ ) separated by 1.5 $\mathrm{mm}$, as for an actual electrode.

The influence of model parameters on signals simulated in both modalities was analyzed qualitatively, by visual analysis of signals, and quantitatively, using the two following criteria.

First, the spike-to-background ratio $(S B R)$ was defined to quantify the influence of the spatial extent and the synchronization degree of the epileptic source on the amplitude of the epileptic spikes appearing in simulated scalp and intracerebral signals. $S B R$ is analogous to the standard signal-to-noise ratio. For each sensor, it was defined as the ratio between the average power of the spike and the average power of the background activity that precedes and follows the spike:

$$
S B R(i)=10 . \log _{10}\left(\frac{n_{b}}{n_{z}} \frac{\sum_{z E_{i}}\left[x_{i}(t)\right]^{2}}{\sum_{z A_{b}}\left[x_{i}(t)\right]^{2}}\right), \quad i=1, \ldots, M
$$

where $x_{i}(t)$ is the electrical potential recorded, at discrete time $t$, at the $i$ th sensor $(i=1, \ldots, M) ; \Delta_{s}\left(\Delta_{b}\right)$ and $n_{s}\left(n_{b}\right)$ denote for the time support and the number of time samples for the spike (the background) respectively. For both scalp and intracerebral EEG signals, $S B R$ values were then averaged over $M$ sensors, leading to a "global" criterion (denoted by an asterisk):

$$
S B R^{*}=\frac{1}{M} \sum_{i=1}^{M} S B R(i)
$$

Second, the normalized root mean square error (nRMSE) was used to evaluate the influence of the location of the epileptic source on the topography of the epileptic spikes observed on simulated scalp and intracerebral signals. For each sensor, $n R M S E$ is a normalized point-to-point distance between a reference signal $\mathbf{x}_{i}$ (EEG potentials recorded at the $i$ th sensor when the epileptic source is located at a 
reference position) and a signal $\mathbf{y}_{i}$ (EEG potentials recorded at the $i$ th sensor when the epileptic source is located at a given position):

$$
n R M S E(i)=\left\{\frac{\sum_{t=1}^{T}\left[x_{i}(t)-y_{i}(t)\right]^{2}}{\sum_{i=1}^{T}\left[x_{i}(t)\right]^{2}}\right\}^{\frac{1}{2}}, \quad i=1, \ldots, M
$$

where $T$ refers to the number of time samples. Similarly, $n R M S E$ values were averaged over $M$ sensors, leading to the average $n R M S E$ (also denoted by an asterisk):

$$
n R M S E^{\star}=\frac{1}{M} \sum_{i=1}^{M} n R M S E(i)
$$

\section{Results}

\section{Scenario 1 - Influence of the spatial extent of the source}

To study the relationship between the spatial extent of the source and the amplitude of the epileptic spikes on simulated scalp and intracerebral EEG signals, we considered an epileptic patch of fixed location (left temporo-parieto-occipital region) and of surface $(S)$ varying from $1 \mathrm{~cm}^{2}$ to $50 \mathrm{~cm}^{2}$ (Fig. 3-a). The same time-course was associated to all current dipoles within this patch. It corresponded to an epileptic spike (see Fig. 2-a) generated by the neuronal population model for an increased excitation-related parameter. The time-course assigned to all other dipoles (outside this patch) corresponded to different realizations of the background activity (see Fig. 2-a) obtained for a "standard" excitation value in the neuronal population model.

Fig. 3-b shows the evolution of the average $S B R$ (denoted by $S B R^{*}$ ) with respect to the patch surface, for scalp and intracerebral EEG signals simulated over the same time epoch. As a first general comment, the $S B R^{*}$ curve was found to be smoother for scalp EEG, compared intracerebral EEG. Particularly, in this second modality, the obtained curve showed local minima $\left(S=11 \mathrm{~cm}^{2}, S=21 \mathrm{~cm}^{2}\right.$ and $S=30 \mathrm{~cm}^{2}$ ). For both modalities, the $S B R^{*}$ increased as the epileptic patch got larger. This increase followed a logarithmic shape, the $S B R^{*}$ increase being less pronounced for larger patch 
surfaces. For a patch surface inferior to about $30 \mathrm{~cm}^{2}$, the $S B R^{*}$ values obtained for simulated intracerebral EEG signals were higher than those obtained for simulated scalp EEG signals. In particular, the characteristic value of $S B R^{*}=3 \mathrm{~dB}$ (corresponding to a spike amplitude equal 1.5 times the amplitude of the surrounding background activity) was obtained for a patch surface of $3 \mathrm{~cm}^{2}$ in the case of intracerebral signals. For this same $S B R^{*}$ value, the patch surface was more than twice as large $\left(7 \mathrm{~cm}^{2}\right)$ in the case of scalp signals. Beyond $30 \mathrm{~cm}^{2}$, the two curves tended to overlap; for a given patch surface, similar values of the $S B R^{*}$ were obtained for scalp and intracerebral data.

The simulated scalp and intracerebral EEG signals for three specific $S B R^{*}$ values $(3,6$ and $9 \mathrm{~dB})$ are illustrated on Figs. 3-c and 3-d. As depicted in Fig. 3-b, these values were obtained for $S=7 \mathrm{~cm}^{2}, S=$ $18 \mathrm{~cm}^{2}, S=32 \mathrm{~cm}^{2}$ (scalp EEG) and for $S=3 \mathrm{~cm}^{2}, S=9 \mathrm{~cm}^{2}$ and $S=24 \mathrm{~cm}^{2}$ (intracerebral EEG). The visual analysis of the scalp EEG signals showed that for $S=7 \mathrm{~cm}^{2}$, spikes were observed on left temporo-parietal electrodes (P7 and T7) "facing” the epileptic patch. As the patch surface increased (S $=18 \mathrm{~cm}^{2}$ ), the spike amplitude was higher on these electrodes (as expected) and a concomitant spike of inverse polarity was clearly visible on parieto-central $(\mathrm{Cz}, \mathrm{Pz})$ and contralateral electrodes $(\mathrm{F} 4, \mathrm{C} 4$, $\mathrm{T} 8, \mathrm{P} 4, \mathrm{P} 8)$. For a surface of $32 \mathrm{~cm}^{2}$, the spike was visible on almost all scalp electrodes.

Simulated intracerebral EEG signals are displayed for each sensor of the intracerebral electrode on Fig. 3-d. For a $3 \mathrm{~cm}^{2}$ patch, the spike appears on the most external sensors (11-15) with a maximum on sensor 12. When $S=9 \mathrm{~cm}^{2}$, the amplitude of the spike increased on sensors 11 to 15 with still a maximum on sensor 12; a small amplitude spike could also be detected on several deeper sensors (710). For a $24 \mathrm{~cm}^{2}$ patch, the spike was visible on 10 adjacent sensors (5-15) with a maximum on sensor 10. It is noteworthy that inversions of the signal phase were also observed along the intracerebral electrode for this higher patch surface. For instance, the phase of the simulated signal was positive on sensor 12 and negative on sensor 10, with an electrical field that almost cancelled at the level of sensor 11 .

\section{Scenario 2 - Influence of the location of the source}


In order to study the influence of the source location on simulated EEG signals, one epileptic patch was positioned in the temporo-parieto-occipital junction (see reference position 0 in Figs. 4-a and 5-a, defined as the intracerebral electrode entry point). This patch was moved along an anterior-posterior (condition 1, Fig. 4-a) and an inferior-superior (condition 2, Fig. 5-a) axis. The patch had a constant surface of $7 \mathrm{~cm}^{2}$, and its barycenter was moved away from the reference position by steps of about 1 $\mathrm{cm}$ along the two axes. At each patch position, signals were simulated on both scalp electrodes and intracerebral electrode sensors, and were quantitatively compared to those obtained when the patch is located at the reference position, using the average $n R M S E\left(n R M S E^{*}\right)$ criterion.

Comparison results are given in Figs. 4-b and 5-b. In both experiments, $n R M S E^{*}$ values computed from intracerebral EEG signals were higher than those computed from scalp EEG signals, except for position -1 (condition 1) and for position +4 (condition 2). The main findings obtained in each condition are described below.

In condition 1, for intracerebral signals, the $n R M S E^{*}$ dramatically increased as soon as the epileptic patch was moved away from the reference position. It remained relatively stable from position +2 to position +7 . For scalp signals, the $n R M S E^{*}$ was found to vary considerably even when the source was moved by one step only. A local minimum was found at position +4 . On the opposite, similar $n R M S E^{*}$ values could be obtained for non adjacent positions (see positions -2 vs $+5,+1$ vs. +4 or +3 vs. +7 , for instance). Simulated intracerebral EEG signals are given in Fig. 4-c, for all patch positions defined in Fig. 4-a. For locations $0,-1$, and -2 , the spikes were well visible on lateral sensors (9 to 15 ) with a maximum on sensor 12 . When the patch was moved anteriorly (positions +1 to +7 ), the spike could not be visually detected anymore. Fig. 4-d displays the simulated scalp voltage maps (63 electrodes), at the time of the maximal spike negativity. It is noteworthy that two adjacent positions, like positions +4 and +5 , could lead to very different maps, while two distant positions like +1 and +4 could disclose relatively similar topographies. Interestingly, for the most anterior positions in the temporal lobe, topographic maps disclosed a "bipolar" voltage distribution whereas for most posterior positions, the maps kept the "radial" aspect obtained at reference position.

In condition 2, simulations led to similar findings, as summarized in Fig. 5. The similarity between maps computed for non-adjacent positions was even more obvious, as compared to condition 1. As an 
example, for positions $-4,-1$ and +1 , we measured comparable $n R M S E^{*}$ values (Fig. 5-b) and found scalp voltage maps were qualitatively similar (Fig. 5-d). A transition from "radial" to "bipolar" voltage distribution was also observed.

\section{Scenario 3 - Influence of the synchronization of the source}

The purpose of this scenario was to study the influence, on the amplitude of simulated epileptic spikes, of the synchronization degree among populations of neurons. We considered a single epileptic patch of $7 \mathrm{~cm}^{2}$ located at the temporo-parieto-occipital junction as depicted in scenario 1 . This patch consisted of $N$ populations of neurons. In order to simulate this patch activity, we used a mixing of $n$ synchronous and (N-n) non-synchronous "epileptic" time-courses (Fig. 6-a). Synchronous timecourses corresponded to the exact same realization of the neuronal population model output, while non-synchronous time-courses corresponded to different realizations (i.e. random time-shift between transient spikes at the population level). The $n$ populations with synchronous activity were randomly chosen according to a uniform distribution. Therefore, the synchronization degree within the patch (percentage $\alpha$ ), was defined as $\alpha=n / N \times 100$. Moreover, for each $\alpha$ varying from 0 to $100 \%$, by step of $10 \%$, an average $S B R^{*}$ value was estimated from signals simulated for 50 different random draws of the $n$ synchronous populations inside the patch, in order to account for the position variability.

The results are displayed in Fig. 6-b. The average of $S B R^{*}$ values (over the 50 random draws) increased both for intracerebral and scalp EEG, as the patch synchronization intensified. The slope of the curve was steeper for intracerebral EEG than for scalp EEG and, from $20 \%$ synchronization, average $S B R^{*}$ values measured for intracerebral signals were found to be higher than those computed on scalp signals. As an example, an average $S B R^{*}$ value of $3 \mathrm{~dB}$ was reached for $\alpha=40 \%$ in intracerebral signals while this same value was reached for $\alpha=78 \%$ in scalp signals. It is noteworthy that for a given synchronization degree, the standard deviation of the average $S B R^{*}$ value was higher for intracerebral EEG than for scalp EEG.

Figs. 6-c and 6-d present examples of simulated scalp and intracerebral EEG signals, respectively. Epileptic spikes became clearly visible for $\alpha=70 \%$ (respectively 40\%) on scalp EEG signals 
(respectively intracerebral EEG signals) and their amplitude gradually increased with the patch synchronisation degree in both cases.

\section{Discussion}

In this study, an attempt was made to quantitatively investigate the relationship between the EEG signals and the spatio-temporal configuration of the underlying neuronal sources. This issue was addressed through a realistic model of EEG generation. The proposed model relies on an accurate representation of the neuronal sources of activity that associates both an anatomically realistic description of the spatial features of the sources (convoluted dipole layer) and a physiologically relevant description of their temporal activities (coupled neuronal populations). Indeed, a better understanding of the generation of electrophysiological signals, both on a spatial and on a temporal point of view, is an essential step towards a better interpretation of the recorded signals.

Our model was used for simulating scalp EEG and intracerebral EEG signals simultaneously, in the particular context of epileptiform activity (transient interictal spikes). Its integrative properties allowed for the bridging between the recorded signals and the spatial and temporal features of the underlying neuronal sources. The relations that we could established between the source-related parameters (spatial extent, location, synchronization) and the simulated EEG signals (amplitude of spikes, amplitude gradient along intracerebral electrodes, topography over electrodes) as well as the limitations of the proposed approach, are discussed in the following paragraphs.

As far as the influence of the spatial extent of the source is concerned, our results show that the cortical area involved in the generation of transient interictal spikes is rather large. Although this result has already been suggested by several studies (Ebersole, 1997; Merlet and Gotman, 1999; Alarcón et al., 2001), we were able to accurately quantify the contribution of a "focal" epileptic source of a given area to the amplitude of resulting spikes observed in EEG signals. In our model, epileptic spikes with amplitude 1.5 times higher than the background activity were obtained for cortical sources of $3 \mathrm{~cm}^{2}$ in the intracerebral EEG and $7 \mathrm{~cm}^{2}$ in the scalp EEG. A better signal-to-noise ratio value of $9 \mathrm{~dB}$, which 
corresponds to spikes with amplitude encompassing 2.8 times the background activity, was reached for an epileptic source area of $24 \mathrm{~cm}^{2}$ and $30 \mathrm{~cm}^{2}$ in the intracerebral and scalp EEG signals, respectively. As expected, these results also illustrate a high sensitivity of the intracerebral EEG, which can detect the activity arising from smaller cortical areas as compared to scalp EEG.

Determining the minimum surface of the cortical sources of scalp-recordable epileptic spikes has been a challenging issue. Most authors who have dealt with this question quoted an early study by Cooper et al. (1965) suggesting that a cortical surface of at least $6 \mathrm{~cm}^{2}$ was necessary to obtain a similar signal attenuation as that observed in the case of a "non-specific" activity on frontal scalp electrodes. It is noteworthy that "non-specific" refers here to a background activity. Therefore the 6 $\mathrm{cm}^{2}$ value is most likely underestimating the cortical area involved in larger transient events like epileptic spikes. Moreover their experiment was not conducted in vivo but on a head phantom (piece of fresh wet skull, a polythene sheet and artificial cortical sources made from pads of cotton wool soaked in saline and connected to a pulse generator). More recent studies based on intracranial recordings (electrocorticography) showed that an epileptic spike is visible on the scalp EEG recording as far as a minimal cortical surface of about $8 \mathrm{~cm}^{2}$ is activated (Ebersole, 1997). The same team reconsidered this estimation and showed that among the 600 intracranial spikes they analyzed, most were visible on scalp EEG recordings when the cortical area ranged between $10 \mathrm{~cm}^{2}$ and $30 \mathrm{~cm}^{2}$ (Tao et al., 2005). Interestingly, this team made a similar observation during ictal periods (Tao et al., 2007a) and suggested that a mean neocortical surface of $19 \mathrm{~cm}^{2}$ was necessary to produce scalp-recordable rhythmic EEG activity at seizure onset. Finally, comparable values were also reported by Kobayashi et al. (2005), who had to consider cortical sources of $6-24 \mathrm{~cm}^{2}$ in the lateral temporal lobe and of $6-30$ $\mathrm{cm}^{2}$ in the frontal lobe, in order to simulate well recordable spikes on scalp EEG. In this last study however, it is noteworthy that the authors did not aim at investigating the relationship between cortical sources and EEG signals, but rather at evaluating the accuracy of different single-dipole localization methods using the simulated EEG signals.

In addition to the spatial extent, the relative location of the source with respect to recording electrodes (scalp and intracerebral) also plays a crucial role in the properties of the recorded signals. From the results obtained in scenario 2, intracerebral EEG recordings appear more highly sensitive to 
the source location than the scalp EEG recordings. Indeed, only the activity of the epileptic sources located in the close neighborhood of the intracerebral electrode was visible in the simulated signals. However, the results also showed that the geometry of the source plays a key role. Indeed, as suggested by the asymmetrical aspect of the $n R M S E^{*}$ curves, two sources located at an equivalent distance from a sensor (like position +1 and -1 ) can generate different sets of intracerebral data. Therefore, according to the spatial configuration of a cortical source, the proper recording of the intracerebral signals can either be impaired even when the source is relatively close (e.g. position +1$)$, or be favored even when the source is more distant (e.g. position -2). The influence of the source geometry is also well illustrated in scenario 1 . As the area of the epileptic patch was progressively increased, the maximum amplitude of the spike initially observed on the lateral sensor of the electrode moved towards a more mesial sensor even if the barycenter of the patch remained the same. In that case, the increase of the spatial extent of the cortical patch most likely implies a modification of its geometry and thus, of its global contribution to the recording sensors.

As far as the scalp EEG signals are concerned, our results showed a lower sensitivity to the source position and consequently to its geometry. Since electrodes are relatively far from brain sources, their volume of sensitivity is greater when compared to intracerebral sensors. This is exemplified in different instances of scenario 2 (like in the case of position -4 and -1 of condition 2) in which different locations of the "focal" epileptic source can lead to relatively similar distribution of electrical potentials on the head. In this scenario, the relatively small area of the considered source $\left(7 \mathrm{~cm}^{2}\right)$ could explain the high variability in the $n R M S E^{*}$ curve encountered for scalp EEG signals. This variability can be explained, at least in part, by the effect of the underlying convoluted cortical anatomy which is more pronounced for small-size patches. This point was verified in scenario 2 as smoother $n R M S E^{*}$ curves could be obtained for larger cortical areas (18 and $32 \mathrm{~cm}^{2}$, not shown).

We also addressed the issue of the degree of synchronization between neuronal populations within the epileptic source. The exact mechanisms leading to abnormal synchronization between populations of neurons over large neocortical surface are not completely understood, particularly in the context of transient spikes or spike-waves observed in partial epilepsy. In this study, we chose to implement a model according to which i) all populations inside the patch are rendered "hyperexcitable" and ii) a 
subset of neuronal populations generate a totally synchronous activity whereas the remaining populations are characterized by asynchronous activity. Therefore, for given patch surface, the key parameter in this model is the fraction of populations (randomly distributed over the patch area) with synchronous activity with respect to the total number of populations. Although relatively simple, our model is in line with the study reported in (Neckelmann et al., 1998). Indeed, authors showed that short distance cortico-cortical linkages in the "epileptic system" play a key role in the initiation and synchronization of paroxysmal activity, although their study was focused on spike-wave complexes and cortically generated seizures in animals.

Results showed clear differences in the influence of the synchronization parameter on simulated intracerebral and scalp EEG signals. On the one hand, scalp-recordable epileptic spikes (with a $S B R^{*}$ higher than $3 \mathrm{~dB}$ ) were obtained for relatively high values of the degree of synchronization $(>70 \%)$. This result corroborates those reported in previous studies suggesting that only widely synchronized cortical activities are observed on the scalp (Cooper et al., 1965). On the other hand, results showed that epileptic spikes corresponding to low synchronization degree (about $40 \%$ ) could be observed in simulated intracerebral EEG signals. It is noteworthy that those spikes were low amplitude and that they could be non-concomitant on adjacent electrode sensors. Therefore, modeling results indicate that intracerebral EEG signals can reflect epileptic activities corresponding to a weak and partial synchronization between neuronal populations forming the epileptic patch.

Although our model provided insights into the relationship between EEG signals and the configuration of the underlying sources of activity, it still suffers from some limitations. Like most physiological models, it can only approximate natural phenomena because the underlying biophysical and biomathematical description is imperfect. In particular, the neuronal population model we used to represent the time-varying intensity of dipoles was reduced to its simpler form (one sub-population of pyramidal cells and one sub-population of local interneurons). In actual neocortex, the cellular organization is obviously more complex. Thus, we are currently working on a more complete model for the neocortical neuronal population. It will integrate other subsets of neurons possibly involved 
into the generation of fast oscillations that are also a marker of epileptogenic processes (Traub et al., 1999).

Another drawback of our model is that it does not account for the contribution of subcortical structures to signals recorded by electrodes. This question, which is still highly debated among the neuroimaging community, is crucial in certain types of epilepsy. For instance, in mesial temporal lobe epilepsy, it is often very difficult to determine whether - and how - epileptic sources located in mesiotemporal structures (like the hippocampus) play a role in visually detectable scalp EEG interictal spikes (Alarcón et al., 1994; Lantz et al., 1997; Merlet et al., 1998; Merlet and Gotman, 1999; Gavaret et al., 2004). Technically, the framework we elaborated to simulate EEG signals could relatively easily account for deep sources, provided that some appropriate information about the spatial features of cellular fields in sub-cortical structures is known.

Our work is a further step in the progress that can still be made in "decoding" the information conveyed by human electromagnetic signals, particularly by better understanding the relationship between the recorded signals and the underlying neuronal activity. Several directions are considered for future work. In particular, we recently extended the model to the generation of magnetoencephalographic (MEG) signals (Badier et al., 2007) which offers a chance to quantitatively explore the complementarity of EEG and MEG modalities. Moreover, since the ground truth is known in the model (number of active sources, location, spatial extent, synchronization level, etc.), it provides an ideal framework to evaluate the many EEG/MEG source localization methods on simulated signals.

The present study reveals the potential value of our model in the particular context of epilepsy. However, this approach could also be adapted to other fields of EEG-based research. In particular, the relationship between the properties of the recorded EEG signals and the location, spatial extent, geometry, synchronization level, and temporal dynamics of the underlying generators is often questioned in EEG rhythms or ERP correlates of cognition.

\section{Acknowledgements}


This work was supported in part by the French Ministry of Research (ACI Neurosciences Intégratives et Computationnelles 2002-2006). 


\section{References}

Abraham, K., Ajmone-Marsan, C.A., 1958. Patterns of cortical discharges and their relation to routine scalp electroencephalography. Electroencephalogr. Clin. Neurophysiol. 10(3), 447-61.

Alarcón, G., Guy, C.N., Binnie, C.D., Walker, S.R., Elwes, R.D., Polkey, C.E., 1994. Intracerebral propagation of interictal activity in partial epilepsy: implications for source localisation. J. Neurol. Neurosurg. Psychiatry 57(4), 435-49.

Alarcón, G., Kissani, N., Dad, M., Elwes, R.D., Ekanayake, J., Hennessy, M.J., Koutroumanidis, M., Binnie, C.D., Polkey, C.E., 2001. Lateralizing and localizing values of ictal onset recorded on the scalp: evidence from simultaneous recordings with intracranial foramen ovale electrodes. Epilepsia 42(11), 1426-37.

Amblard, C., Lapalme, E., Lina, J.M., 2004. Biomagnetic source detection by maximum entropy and graphical models. IEEE Trans. Biomed. Eng. 51(3), 427-42.

Badier, J.M., Cosandier-Rimélé, D., Bénar, C.G., Schwartz, D., Chauvel, P., Wendling, F., 2007. Realistic synthetic background neuronal activity for the analysis of MEG probe configurations. Conf. Proc. IEEE Eng. Med. Biol. Soc. 1, 2460-3.

Baillet, S., Garnero, L., 1997. A Bayesian approach to introducing anatomo-functional priors in the EEG/MEG inverse problem. IEEE Trans. Biomed. Eng. 44(5), 374-85. 
Cooper, R., Winter, A.L., Crow, H.J., Walter W.G., 1965. Comparison of subcortical, cortical and scalp activity using chronically indwelling electrodes in man. Electroencephalogr. Clin. Neurophysiol. $18,217-28$.

Cosandier-Rimélé, D., Badier, J.M., Chauvel, P., Wendling, F., 2007. A physiologically plausible spatio-temporal model for EEG signals recorded with intracerebral electrodes in human partial epilepsy. IEEE Trans. Biomed. Eng. 54(3), 380-8.

Dale, A.M., Sereno, M.I., 1993. Improved localization of cortical activity by combining EEG and MEG with MRI cortical surface reconstruction: a linear approach. J. Cogn. Neurosci. 5, 162-76.

Daunizeau, J., Mattout, J., Clonda, D., Goulard, B., Benali, H., Lina, J.M., 2006. Bayesian spatiotemporal approach for EEG source reconstruction: conciliating ECD and distributed models. IEEE Trans. Biomed. Eng. 53(3), 503-16.

Ebersole, J.S., 1997. Defining epileptogenic foci: past, present, future. J. Clin. Neurophysiol. 14(6), $470-83$.

Freeman, W.J., 1978. Models of the dynamics of neural populations. Electroencephalogr. Clin. Neurophysiol. Suppl. (34), 9-18.

Gavaret, M., Badier, J.M., Marquis, P., Bartolomei, F., Chauvel, P., 2004. Electric source imaging in temporal lobe epilepsy. J. Clin. Neurophysiol. 21(4), 267-82.

Geisler, C.D., Gerstein, G.L., 1961. The surface EEG in relation to its sources. Electroencephalogr. Clin. Neurophysiol. 13, 927-34. 
Gloor, P., 1985. Neuronal generators and the problem of localization in electroencephalography: application of volume conductor theory to electroencephalography. J. Clin. Neurophysiol. 2(4), 32754.

Gonçalves, S.I, de Munck, J.C., Verbunt, J.P., Bijma, F., Heethaar, R.M., Lopes da Silva, F., 2003. In vivo measurement of the brain and skull resistivities using an EIT-based method and realistic models for the head. IEEE Trans. Biomed. Eng. 50(6), 754-67.

Gorodnitsky, I.F., George, J.S., Rao, B.D., 1995. Neuromagnetic source imaging with FOCUSS: a recursive weighted minimum norm algorithm. Electroencephalogr. Clin. Neurophysiol. .95(4), 231-51.

Hämäläinen, M.S., Hari, R., Ilmoniemi, R.J., Knuutila, J., Lounasmaa, O.V., 1993. Magnetoencephalography - theory, instrumentation and applications to noninvasive studies of the working human brain. Rev. Mod. Phys. 65, 413-98.

Hämäläinen, M.S., Ilmoniemi, R.J., 1994. Interpreting magnetic fields of the brain: minimum norm estimates. Med. Biol. Eng. Comput. 32(1), 35-42.

Hämäläinen, M.S., Sarvas, J., 1989. Realistic conductivity geometry model of the human head for interpretation of neuromagnetic data. IEEE Trans. Biomed. Eng. 36(2), 165-71.

Jansen, B.H., Rit, V.G., 1995. Electroencephalogram and visual evoked potential generation in a mathematical model of coupled cortical columns. Biol. Cybern. 73(4), 357-66.

Kobayashi, K., Yoshinaga, H., Ohtsuka, Y., Gotman, J., 2005. Dipole modeling of epileptic spikes can be accurate or misleading. Epilepsia 46(3), 397-408. 
Lantz, G., Ryding, E., Rosén, I., 1997. Dipole reconstruction as a method for identifying patients with mesolimbic epilepsy. Seizure 6(4), 303-10.

Lopes da Silva, F.H., van Rotterdam, A., Barts, P., van Heusden, E., Burr, W., 1976. Models of neuronal populations: the basic mechanisms of rhythmicity. Prog. Brain Res. 45, 281-308.

Mangin, J.F., Frouin, V., Bloch, I., Régis, J., Lopez-Krahe, J., 1995. From 3D Magnetic resonance images to structural representations of the cortex topography using topology preserving deformations. J. Math. Imag. Vision. 5, 297-318.

Meijs, J.W., Weier, O.W., Peters, M.J., van Oosterom, A., 1989. On the numerical accuracy of the boundary element method. IEEE Trans. Biomed. Eng. 36(10), 1038-49.

Merlet, I., Garcia-Larrea, L., Ryvlin, P., Isnard, J., Sindou, M., Mauguière, F., 1998. Topographical reliability of mesio-temporal sources of interictal spikes in temporal lobe epilepsy. Electroencephalogr. Clin. Neurophysiol. 107(3), 206-12.

Merlet, I., Gotman, J., 1999. Reliability of dipole models of epileptic spikes. Clin. Neurophysiol. $110(6), 1013-28$.

Neckelmann D., Amzica F., Steriade M., 1998. Spike-Wave Complexes and Fast Components of Cortically Generated Seizures. III. Synchronizing Mechanisms. J. Neurophysiol. 80, 1480-1494.

Pascual-Marqui, R.D., Michel, C.M., Lehmann, D., 1994. Low resolution electromagnetic tomography: a new method for localizing electrical activity in the brain. Int. J. Psychophysiol. 18(1), $49-65$ 
Plonsey, R., Heppner, D.B., 1967. Considerations of quasi-stationarity in electrophysiological systems. Bull. Math. Biophys. 29(4), 657-64.

Suffczynski, P., Kalitzin, S., Lopes da Silva, F.H., 2004. Dynamics of non-convulsive epileptic phenomena modeled by a bistable neuronal network. Neuroscience 126(2), 467-84.

Suffczynski, P., Wendling, F., Bellanger, J.J., Lopes da Silva, F.H., 2006. Some insights into computational models of (patho)physiological brain activity. Proc. IEEE 94(4), 784-804.

Tao, J.X., Ray, A., Hawes-Ebersole, S., Ebersole, J.S., 2005. Intracranial EEG substrates of scalp EEG interictal spikes. Epilepsia 46(5), 669-76.

Tao, J.X., Baldwin, M., Ray, A., Hawes-Ebersole, S., Ebersole, J.S., 2007a. The impact of cerebral source area and synchrony on recording scalp electroencephalography ictal patterns. Epilepsia 48(11), 2167-76.

Tao, J.X., Baldwin, M., Hawes-Ebersole, S., Ebersole, J.S., 2007b. Cortical substrates of scalp EEG epileptiform discharges. J. Clin. Neurophysiol. 24(2), 96-100.

Traub, R.D., 1979. Neocortical pyramidal cells: a model with dendritic calcium conductance reproduces repetitive firing and epileptic behavior. Brain Res. 173(2), 243-57.

Traub, R.D., Jefferys, J.G.R., Whittington, M.A., 1999. Fast oscillations in cortical circuits. MIT Press, Cambridge, MA.

Wendling, F., Bellanger, J.J., Bartolomei, F., Chauvel, P., 2000. Relevance of nonlinear lumpedparameter models in the analysis of depth-EEG epileptic signals. Biol. Cybern. 83(4), 367-78. 
Wendling, F., Hernandez, A., Bellanger, J.J., Chauvel, P., Bartolomei, F., 2005. Interictal to ictal transition in human temporal lobe epilepsy: insights from a computational model of intracerebral EEG. J. Clin. Neurophysiol. 22(5), 343-56.

Wendling, F., Chauvel, P., 2008. Transition to ictal activity in temporal lobe epilepsy: insights from macroscopic models. In: Soltesz I. and Staley K. (Eds.), Computational neuroscience in epilepsy, in press.

Wilson, H.R., Cowan, J.D., 1972. Excitatory and inhibitory interactions in localized populations of model neurons. Biophys. J. 12(1), 1-24.

Zanow, F., Peters, M.J., 1995. Individually shaped volume conductor models of the head in EEG source localization. Med. Biol. Eng. Comput. 33(4), 582-8. 


\section{Figure legends}

Fig. 1: Architecture of the spatio-temporal extended source model. a) A triangular mesh of the cortical surface (grey-white matter interface) is built from the segmentation of 3D MRI data and is composed of small elementary triangles $\left(\sim 1 \mathrm{~mm}^{2}\right.$ per triangle). b) Each triangle is assumed to represent a neuronal population. The electrical contribution of each neuronal population is represented by an elementary current dipole, placed at the barycentre of the triangle and oriented orthogonally to triangle surface. The moment of each current dipole is weighted by both the triangle area and the population time-varying field activity. c) This field activity is obtained from a model of coupled neuronal populations. Each neuronal population is made of two subsets of neurons: main cells (i.e. pyramidal cells) and local interneurons (i.e. other non-pyramidal cells: stellate or basket cells, etc.). Pyramidal cells receive excitatory input (exc) from other pyramidal cells (collateral excitation) and inhibitory input (inh) from interneurons. These latter cells receive excitatory input only from pyramidal cells.

Fig. 2: Model parameter sensitivity analysis. a) The epileptic source was simulated using a neocortical patch. We assigned to all current dipoles inside the patch an epileptic time-course (interictal spike). All other dipoles, outside this patch, were assigned a "normal" time-course (background activity). b) Scalp EEG signals were computed over 63 scalp electrode (10-10 system). c) Intracerebral EEG signals were computed at a single intracerebral electrode over 15 cylindrical sensors (length: $2 \mathrm{~mm}$, diameter: 0.8 $\mathrm{mm}, 1.5 \mathrm{~mm}$ apart). Sensors are numbered from 1 to 15 , number 1 corresponding to the deepest sensor. The intracerebral electrode was orthogonally implanted in the left temporo-parieto-occipital junction (the yellow circle represents the electrode entry point). On the right, the electrode trajectory is reported on a MRI coronal view.

Fig. 3: Scenario 1 -influence of the source spatial extent. a) Illustration of the epileptic patch located in left temporo-parieto-occipital region, with a surface $(S)$ varying from $1 \mathrm{~cm}^{2}$ to $50 \mathrm{~cm}^{2}$. b) Evolution 
of the average $S B R\left(S B R^{*}\right)$ with respect to the surface of the epileptic patch, for scalp EEG signals (black line) and for intracerebral EEG signals (grey line). c) Examples of simulated scalp EEG signals for three surfaces $\left(7,18\right.$ and $\left.32 \mathrm{~cm}^{2}\right)$. For visualization purposes, only 19 from the 63 channels are presented (10-20 standard system). d) Examples of simulated intracerebral EEG signals for three surfaces $\left(3,9\right.$ and $\left.24 \mathrm{~cm}^{2}\right)$.

Fig. 4: Scenario 2 - influence of the source location, condition 1. a) Illustration of the 10 epileptic patch positions arbitrarily defined along a temporo-occipital line (examples of corresponding epileptic patches are given for position $0,+1,+3,+5$, and +7 ). Reference position 0 corresponded to the intracerebral electrode entry point. At each position, the patch surface is unchanged $\left.\left(7 \mathrm{~cm}^{2}\right) . \mathrm{b}\right)$ Evolution of the average $n R M S E$ (nRMSE ${ }^{*}$ ) with respect to the position of the epileptic patch, for scalp EEG signals (black line) and for intracerebral EEG signals (grey line). c) Simulated intracerebral EEG signals for all 10 source positions. d) Simulated scalp voltage maps (over the 63 scalp EEG electrodes) for all 10 source positions, at the time of the maximal spike negativity. Blue indicates negative values and red indicates positive values.

Fig. 5: Scenario 2 - influence of the source location, condition 2. a) Illustration of the 9 epileptic patch positions arbitrarily defined along an inferio-superior line (examples of corresponding epileptic patches are given for position $-4,-1,0,+1$, and +4$)$. As in condition 1 , reference position 0 corresponded to the intracerebral electrode entry point and the patch surface is unchanged $\left.\left(7 \mathrm{~cm}^{2}\right) . \mathrm{b}\right)$ Evolution of the $n R M S E^{*}$ (nRMSE averaged over sensors) with respect to the position of the epileptic patch, for scalp EEG signals (black line) and for intracerebral EEG signals (grey line). c) Simulated intracerebral EEG signals for all 9 source positions. d) Simulated scalp voltage maps (over the 63 scalp EEG electrodes) for all 9 source positions, at the time of the maximal spike negativity.

Fig. 6: Scenario 3 - influence of the source synchronization degree. a) Illustration of the synchronization degree $(\alpha)$ of the epileptic patch. Percentage $\alpha$ is defined as the proportion, inside the patch, of neuronal populations with synchronous epileptic activity (black dots) vs. non-synchronous 
epileptic activity (white dots). b) Evolution of the average $S B R^{*}\left(S B R^{*}\right.$ averaged over 50 random draws) with respect to the synchronization degree of the epileptic patch, for scalp EEG signals (black line) and for intracerebral EEG signals (grey line). For each point, the bar indicates the standard deviation obtained for 50 random draws. c) Examples of simulated scalp EEG signals for three synchronization degrees (0, 70 and 100\%). d) Examples of simulated intracerebral EEG signals for three synchronization degrees $(0,40$ and $100 \%)$. 
Figure 1

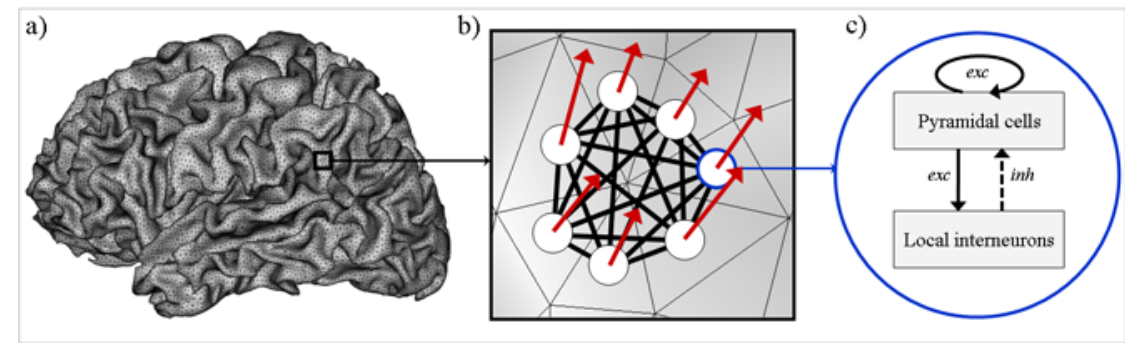

Figure 2
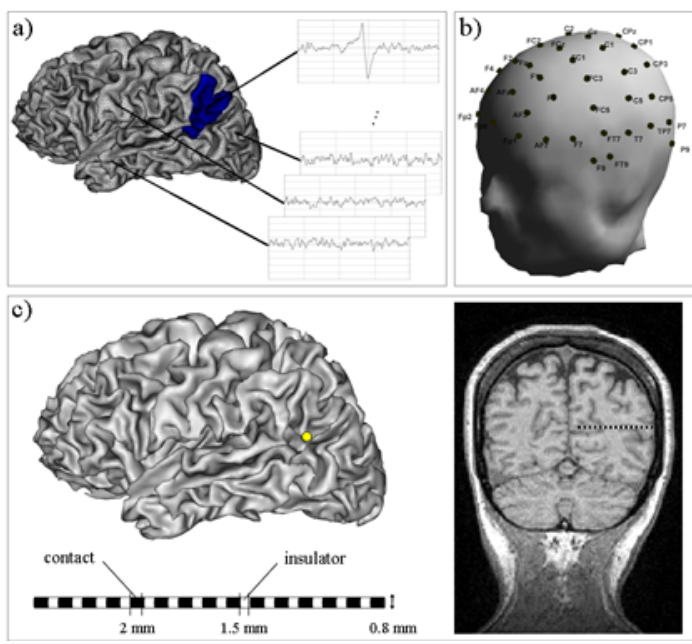

Figure 3

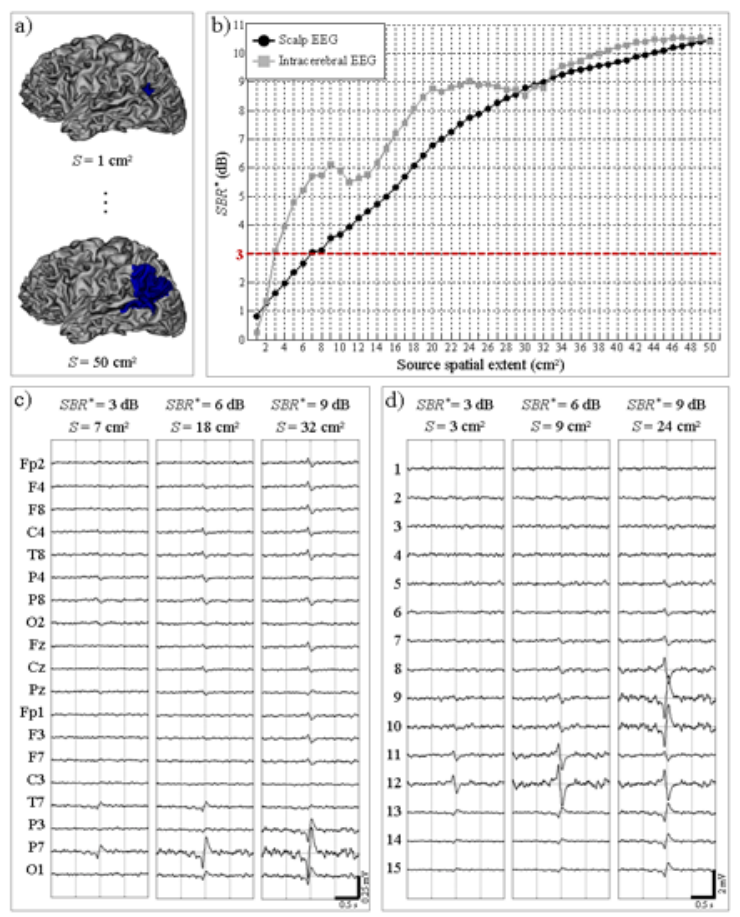


Figure 4

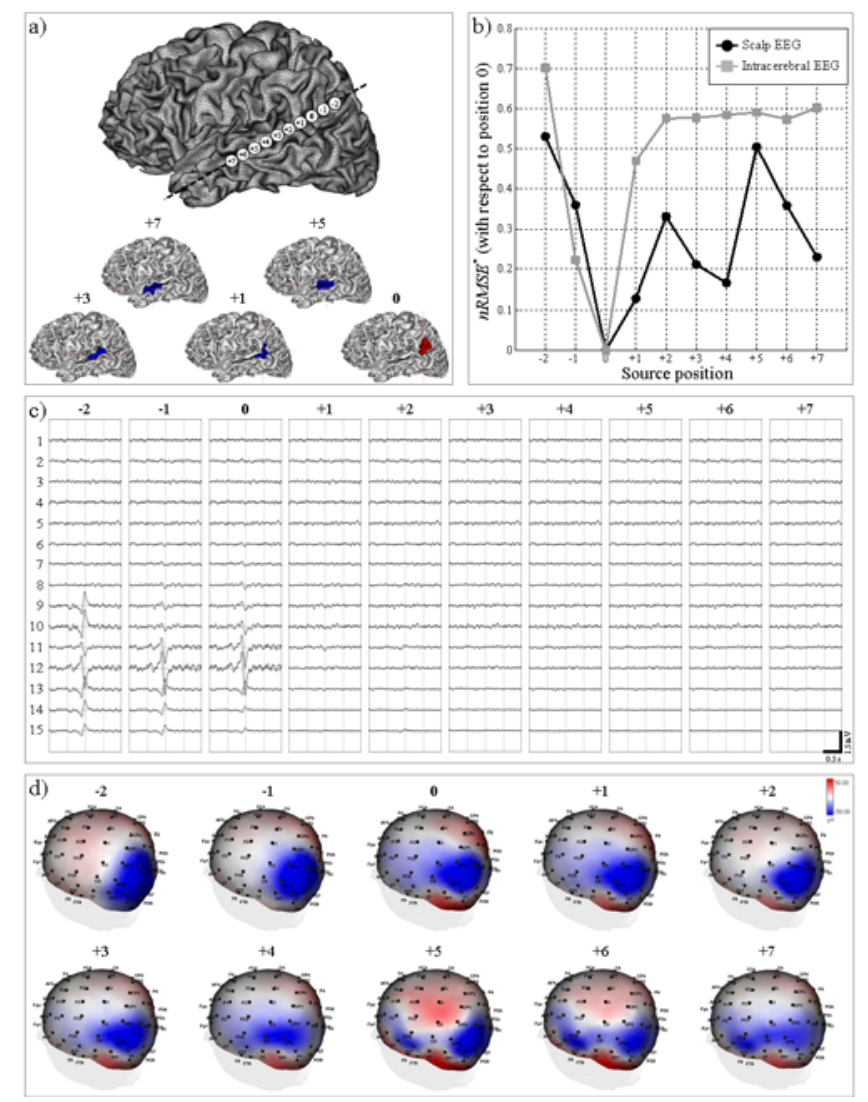

Figure 5

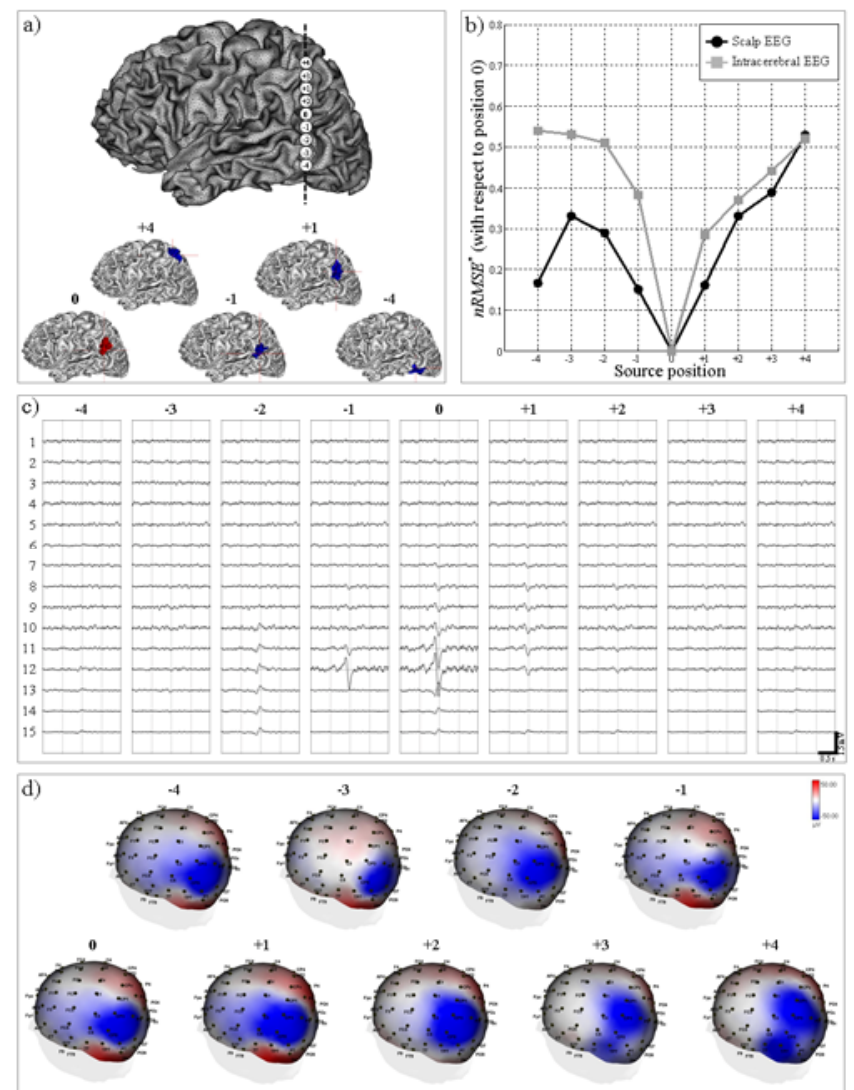


Figure 6

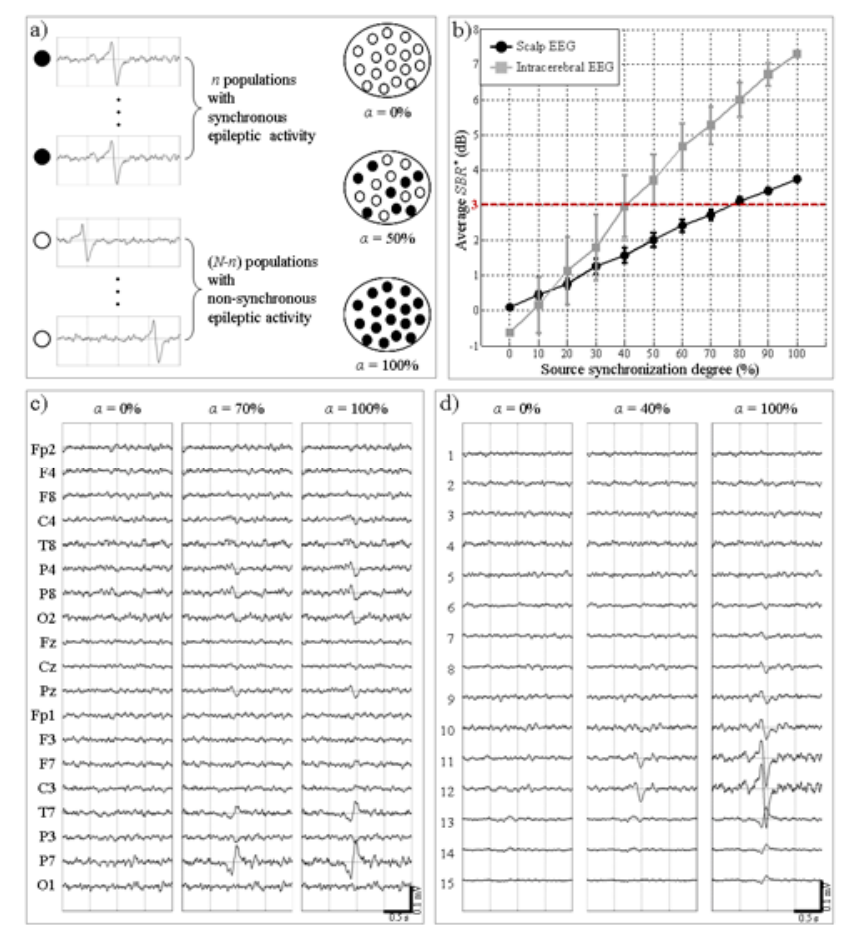

